Research Article

\title{
Stability Analysis of a Class of Neural Networks with State-Dependent State Delay
}

\author{
Yue Chen and Jin-E Zhang $\mathbb{1}$ \\ Hubei Normal University, Huangshi 435002, China \\ Correspondence should be addressed to Jin-E Zhang; zhang86021205@163.com
}

Received 13 February 2020; Accepted 2 April 2020; Published 1 May 2020

Academic Editor: Jianquan Lu

Copyright (c) 2020 Yue Chen and Jin-E Zhang. This is an open access article distributed under the Creative Commons Attribution License, which permits unrestricted use, distribution, and reproduction in any medium, provided the original work is properly cited.

\begin{abstract}
The differential equations with state-dependent delay are very important equations because they can describe some problems in the real world more accurately. Due to the complexity of state-dependent delay, it also brings challenges to the research. The value of delay varying with the state is the difference between state-dependent delay and time-dependent delay. It is impossible to know exactly in advance how far historical state information is needed, and then the problem of state-dependent delay is more complicated compared with time-dependent delay. The dominating work of this paper is to solve the stability problem of neural networks equipped with state-dependent state delay. We use the purely analytical method to deduce the sufficient conditions for local exponential stability of the zero solution. Finally, a few numerical examples are presented to prove the availability of our results.
\end{abstract}

\section{Introduction}

Neural network is an information processing system to simulate the structures and functions of the human brain. As far as we know, the functions and structures of the individual neuron are simple, but the dynamic behavior of the neural network is very rich and intricate $[1,2]$. In the neural network, several simple processing units are connected to each other in a certain way. Neural network system is highly complex, which not only has the common characteristics of general nonlinear systems but also has its own unique features [3-6]. For example, it is proved that the neural network has the ability to approximate nonlinear mapping, and any continuous nonlinear function mapping can be approximated by the multilayer neural network with arbitrary precision. It shows that the neural network has a good application prospect in the challenging nonlinear control field [3]. Also, the parallel distributed integrated optimization processing of information makes the neural network very suitable for solving large-scale real-time computing problems in system control [4]. At the same time, some neural network models have the trait of automatically searching the extremum of energy function, which is very useful in adaptive control design [5]. In addition, the neural network also has the peculiarity of high fault-tolerant ability, generalization ability, and adaptive ability for learning behavior [6].

Over that seventy years, neural networks have been involved in combinatorial optimization, pattern recognition, image processing, robot control, signal processing, and other scientific fields and achieved extensive success [7-10]. In the application of the neural network, on the one hand, information transmission between neurons needs time. On the other hand, due to the influence of hardware implementation in reality (such as limited switching speed), the time delay phenomenon is inevitable [11]. The existence of time delay may induce instability, oscillation, and poor performance, but we can also overcome the unfavorable effect about some types of time delays by developed control frameworks, such as based on the Halanay inequality framework $[1,2,11,12]$. At present, many different delays have been applied to the neural network [2, 12-18], for instance, time-varying delay [12, 14], distributed delay $[13,15,16]$, discrete constant delay $[2,13]$, proportional delay [17], and unbounded delay [18]. 
State-dependent delay (SDD) is all around us [19-22]. With limited natural resources, Antarctic whales and seals tend to mature longer if their populations are large [19]. In the problem of car following, it is inevitable to encounter the phenomenon that the time delay changes with the state, which contains physiological time delay, mechanical time delay, and motion time delay $[20,21]$. In addition, in the blood circulation system, the concentration of nutrients regulates the mitotic cycle of hematopoietic stem cells; thus, the mitotic cycle of stem cells is affected by the concentration of cells in the region [22]. In these cases, in order to describe change and evolution of things more accurately and make the research results more realistic and modest with nuanced understanding, we must adopt differential equations with SDD.

Neurodynamics help to identify the highly complex and precise multilevel nonlinear brain system. The processing of neural information involves the coupling and cooperation of multiple levels and regions. In this way, the nerve activity about the structural neural network is worthy of study and discussion from the perspective of models and evolution, and then to some extent, the cognitive function of the specific functional neural network is realized by evolutive neurodynamics [13]. The work about evolutive neurodynamics will be helpful to understand the information processing mechanism and the neural energy coding rule in the nervous system and also provides the basis for the research of the potential mechanism of cognitive function.

In this paper, the local evolution characteristics of neural networks with state-dependent state delay (SDSD) will be discussed. The topic introduced here may interest researchers in engaging the theory and application of the new neural network model having kinematics and dynamics feature. Prior to this, there have been many studies on the stability of nonlinear systems with SDSD. Hartung [23] described a type of nonlinear functional differential equation with SDSD and analyzed the stability conditions of periodic solutions based on the linearization method. Exponential stability conditions of nonlinear systems with SDSD were reported via comparing with time-dependent delay systems in [24]. Fiter and Fridman [25] developed the Lyapunov-Krasovskii functional method to discuss asymptotic stability about some particular linear systems with SDSD. Li and $\mathrm{Wu}$ [26] considered a class of nonlinear differential systems with SDD pulses. By pulse control theory, uniformly stable, uniformly asymptotically stable, and exponentially stable results were presented. To derive stability criteria of nonlinear systems with SDSD, Li and Yang [27] initially created purely analytical frame structure. To the best of the authors' knowledge, although the neural networks are widely used and the theoretical results are abundant, the research on stability of the neural network with SDSD is still blank. Moreover, since time delay is an inevitable factor in neural networks, it is of great significance to study the problem of neural networks with SDSD. Then, the primary contributions of this paper are generalized as follows: (1) A general neural network model with SDSD is established. Neural network model with SDSD may interest all those professionals and academics in processing operations who would desire to utilize the capabilities of control systems about capturing rich history information for cost effective and yet robust events to be portrayed. Such research also contributes to reveal influences of neurodynamics evoked by SDSD characteristics. (2) Locally exponentially stable sufficient conditions of the neural networks with SDSD are obtained. The purely analytical method employed by us demonstrates that it is possible to analyze computational neurodynamics without a few additional restrictions. The purely analytical method itself intents to address the universality of analysis framework. Definitely, it may be extended to a more general class of nonlinear systems with SDSD.

The rest of this paper is arranged as follows. In Section 2 , we will present a specific neural network model. The results of our work are described in Section 3. In Section 4, two numerical examples and simulation results are given to verify the validity of our results. Finally, in Section 5, the thesis is summarized, and the future work is prospected.

\section{Preliminaries and Model Description}

2.1. Notations. Let $\mathscr{R}$ and $\mathscr{R}^{+}$represent the sets of real numbers and nonnegative real numbers, respectively. $\mathscr{R}^{n}$ denotes the $n$-dimension Euclidean space. For a matrix $E$, $\lambda_{\max }(E)$ is used to denote its maximum eigenvalue. $\mathscr{P}(E)$ stands for the minimum value of all elements of matrix $E$. The vector 1-norm and 2-norm are severally expressed by $\|\cdot\|_{1}$ and $\|\cdot\|_{2}$.

2.2. Some Preliminaries and Problem Formulation. Based on the work of Li and Yang [27], we put forward the following neural network model with SDSD, which is described by

$$
\begin{aligned}
\dot{x}_{i}(t)= & -a_{i} x_{i}(t)+\sum_{j=1}^{n} b_{i j} g_{j}\left(x_{j}(t)\right) \\
& +\sum_{j=1}^{n} d_{i j} f_{j}\left(x_{j}(t-\tau(t, \mathscr{X}))\right), \quad i=1,2, \ldots, n, t \geq t_{0},
\end{aligned}
$$

for the sake of presentation; we also give the compact form of system (1) as follows:

$$
\dot{X}(t)=-A \mathscr{X}(t)+B g(\mathscr{X}(t))+D f(\mathscr{X}(t-\tau(t, \mathscr{X}))),
$$

where $n$ stands for the number of neurons in the network, $\dot{X}(t)$ denotes the upper right derivative of $\mathscr{X}(t)$, $\mathscr{X}=\mathscr{X}(t)=\left(x_{1}(t), x_{2}(t), \ldots, x_{n}(t)\right)^{T}$, and $x_{i}(t)$ represents the state of the $i$ th neuron. $A$ is a diagonal matrix, for $i=1,2, \ldots, n, a_{i}>0$, and $B$ and $D$ are constant matrices with corresponding dimensions. $g(\mathscr{X}(t))=\left(g_{1}\left(x_{1}\right)(t)\right.$, $\left.g_{2}\left(x_{2}(t)\right), \ldots, g_{n}\left(x_{n}(t)\right)\right)^{T}$ and $f(\mathscr{X}(t-\tau(t, \mathscr{X})))=$ $\left(f_{1}\left(x_{1}(t-\tau(t, \mathscr{X}))\right), f_{2}\left(x_{2}(t-\tau(t, \mathscr{X}))\right), \ldots, f_{n}\left(x_{n}(t-\right.\right.$ $\tau(t, \mathscr{X}))))^{T}$ are the excitation functions of the $i$ th neuron at time $t$ and $t-\tau(t, \mathscr{X})$, respectively. 
Furthermore, we use $\mathscr{X}(s)=\Psi(s), s \in\left[t_{0}-\eta, t_{0}\right]$ to denote the initial value of system (2), where $\Psi=\Psi(s)=$ $\left(\psi_{1}(s), \psi_{2}(s), \ldots, \psi_{n}(s)\right)^{T} \in \mathscr{C}\left(\left[t_{0}-\eta, t_{0}\right], \mathscr{R}^{n}\right)$.

$\mathscr{C}\left(\left[t_{0}-\eta, t_{0}\right], \mathscr{R}^{n}\right)$ is a Banach space whose elements are continuous vector-valued functions. These continuous functions map the interval $\left[t_{0}-\eta, t_{0}\right]$ into $\mathscr{R}^{n}$. Let $\|\Psi\|_{\alpha}=$ $\sup _{t_{0}-\eta \leq s \leq t_{0}}\|\Psi(s)\|$ stand for the norm of a function $\Psi(\cdot) \in \mathscr{C}\left(\left[t_{0}-\eta, t_{0}\right], \mathscr{R}^{n}\right)$, where $\|\cdot\|$ is the vector norm matching with the content of the paper.

Remark 1. $\mathscr{X}(t)$ is right-upper derivable, which implies that the solution of system (2) can be continuous but not smooth. The state delay $\tau(t, \mathscr{X})$ is related to the state of each neuron.

For subsequent analysis, we need the following assumptions for system (1) and (2).

Assumption 1. Functions $g(\cdot), f(\cdot) \in \mathscr{R}^{n}$ satisfy $f(0)=0$, $g(0)=0$.

Through Assumption 1, this ensures that $\mathscr{X}=0$ is a constant solution of systems (1) and (2).

Assumption 2. $g(\cdot), f(\cdot) \in \mathscr{R}^{n}$ are locally Lipschitz continuous; in other words, $\forall \beta_{1}, \beta_{2} \in \mathscr{R}$, and we have

$$
\begin{aligned}
& \left|g_{i}\left(\beta_{1}\right)-g_{i}\left(\beta_{2}\right)\right| \leq \ell_{i}\left|\beta_{1}-\beta_{2}\right|, \quad \forall i \in\{1,2, \ldots, n\}, \\
& \left|f_{i}\left(\beta_{1}\right)-f_{i}\left(\beta_{2}\right)\right| \leq \tilde{\ell}_{i}\left|\beta_{1}-\beta_{2}\right|, \quad \forall i \in\{1,2, \ldots, n\},
\end{aligned}
$$

where $\ell_{i}>0$ and $\tilde{\ell}_{i}>0$.

According to Assumption 2, we can get two constant sets $\left\{\ell_{1}, \ell_{2}, \ldots, \ell_{n}\right\}$ and $\left\{\tilde{\ell}_{1}, \tilde{\ell}_{2}, \ldots, \tilde{\ell}_{n}\right\}$. Let $\left\{\ell_{1}, \ell_{2}, \ldots, \ell_{n}\right\}$ and $l_{f}=\max \left\{\widetilde{\ell}_{1}, \widetilde{\ell}_{2}, \ldots, \tilde{\ell}_{n}\right\}$.

Assumption 3. The state delay $\tau(t, \mathscr{X}) \in C\left(\mathscr{R}^{+} \times \mathscr{R}^{n},[0, \eta]\right)$ is locally Lipschitz continuous, namely, for any $\Gamma_{1}, \Gamma_{2} \in \mathscr{R}^{n}$, there always exists a constant $\ell_{\tau}>0$ such that

$$
\left|\tau\left(t, \Gamma_{1}\right)-\tau\left(t, \Gamma_{2}\right)\right| \leq \ell_{\tau}\left\|\Gamma_{1}-\Gamma_{2}\right\|
$$

Assumption 4. When $\mathscr{X}=0, \tau(t, \mathscr{X})$ has supremum equipped with $\tau_{0}(\leq \eta), \sup \left\{\tau(t, 0), t \geq t_{0}\right\}=\tau_{0}$.

For ease of expression, let

$$
\begin{aligned}
& \pi_{1}=\max _{1 \leq i \leq n}\left(-a_{i}+\sum_{j=1}^{n}\left|b_{j i}\right| \ell_{i}\right), \\
& \pi_{2}=\max _{1 \leq i \leq n} \sum_{j=1}^{n}\left|d_{j i}\right| \tilde{\ell}_{i} .
\end{aligned}
$$

Definition 1 (see [28]). The zero solution of system (2) is said to be locally exponentially stable (LES) in region $\mathscr{M}$; if there exist constants $\gamma>0$ and Lyapunov exponent $\zeta>0$, for any $t \geq t_{0}$, we have

$$
\left\|\mathscr{X}\left(t ; t_{0}, \Psi\right)\right\| \leq \gamma\|\Psi\|_{\alpha} e^{-\zeta\left(t-t_{0}\right)}
$$

where $\mathscr{X}\left(t ; t_{0}, \Psi\right)$ is a solution of system (2) with the initial condition $\Psi \in \mathscr{C}\left(\left[t_{0}-\eta, t_{0}\right], \mathscr{M}\right), \mathscr{M} \subset \mathscr{R}^{n}$, and $\mathscr{M}$ is called a local exponential attraction set of the zero solution.

Lemma 1 (see [29]). Let $\Gamma_{1}, \Gamma_{2} \in \mathscr{R}^{n}$, and we have

$$
\Gamma_{1}^{T} \Gamma_{2}+\Gamma_{2}^{T} \Gamma_{1} \leq \omega \Gamma_{1}^{T} \Gamma_{1}+\omega^{-1} \Gamma_{2}^{T} \Gamma_{2},
$$

for any $\omega>0$.

\section{Main Results}

Theorem 1. Under Assumptions 1-4, the zero equilibrium of system (2) is LES if

$$
\pi_{1}+\pi_{2}<0
$$

and Lyapunov exponent $\zeta>0$ satisfies

$$
\zeta+\pi_{1}+\pi_{2} e^{\zeta\left(\ell_{\tau}\|\Psi\|_{\alpha}+\tau_{0}\right)} \leq 0 .
$$

Proof. We assume that $\mathscr{X}\left(t ; t_{0}, \Psi\right)$ is a trajectory of system (2) with initial value $\left(t_{0}, \Psi\right)$, where $\Psi \in \mathscr{C}\left(\left[t_{0}-\eta, t_{0}\right], \mathscr{R}^{n}\right)$ and $\Psi \neq 0$. For the sake of convenience, let $V(t)=V(t, \mathscr{X})=$ $\|\mathscr{X}(t)\|_{1}=\sum_{i=1}^{n}\left|x_{i}(t)\right|$ and $V_{0}=\left\{\sup V(s), s \in\left[t_{0}-\eta, t_{0}\right]\right\}$. Then, for any $\epsilon \in(0, \zeta)$, we claim that

$$
e^{(\zeta-\epsilon)\left(t-t_{0}\right)} V(t) \leq V_{0}, \quad \forall t \geq t_{0}
$$

Firstly, when $t=t_{0}$, (10) holds. Next, we prove that (10) holds on $\left(t_{0},+\infty\right)$. In contrast to $(10)$, there are some instants on $\left(t_{0},+\infty\right)$ to make (10) untenable, and then we can find an instant $t_{q} \geq t_{0}$; the following three events will happen:

(1) $e^{(\zeta-\epsilon)\left(t_{q}-t_{0}\right)} V\left(t_{q}\right)=V_{0}$.

(2) $e^{(\zeta-\epsilon)\left(t-t_{0}\right)} V(t) \leq V_{0}$, for $\forall t \in\left[t_{0}-\eta, t_{q}\right]$.

(3) There exists a right neighbor of $t_{q}\left(U_{+}^{0}\left(t_{q}, \xi\right)\right)$ such that $\forall t^{\xi} \in U_{+}^{0}\left(t_{q}, \xi\right)$ and $e^{(\zeta-\epsilon)\left(t^{\xi}-t_{0}\right)} V\left(t^{\xi}\right)>V_{0}$.

On the contrary, by Assumptions 1-4 and combining (2), the derivative of $e^{(\zeta-\epsilon)\left(t-t_{0}\right)} V(t)$ at time $t_{q}$ is as follows: 


$$
\begin{aligned}
& \left.\frac{\mathrm{d}}{\mathrm{d} t}\left(e^{(\zeta-\epsilon)\left(t-t_{0}\right)} V(t)\right)\right|_{t=t_{q}} \\
& =(\zeta-\epsilon) e^{(\zeta-\epsilon)\left(t_{q}-t_{0}\right)} V\left(t_{q}\right)+\left.\left[e^{(\zeta-\epsilon)\left(t-t_{0}\right)} \dot{V}(t)\right]\right|_{t=t_{q}} \\
& \leq(\zeta-\epsilon) V_{0}+e^{(\zeta-\epsilon)\left(t_{q}-t_{0}\right)}\left(\sum_{i=1}^{n} \operatorname{sgn}\left(x_{i}\left(t_{q}\right)\right)\left(\dot{x}_{i}\left(t_{q}\right)\right)\right) \\
& =(\zeta-\epsilon) V_{0}+e^{(\zeta-\epsilon)\left(t_{q}-t_{0}\right)}\left(\sum _ { i = 1 } ^ { n } \operatorname { s g n } ( x _ { i } ( t _ { q } ) ) \left[-a_{i} x_{i}\left(t_{q}\right)\right.\right. \\
& \left.\left.+\sum_{j=1}^{n} b_{i j} g_{j}\left(x_{j}\left(t_{q}\right)\right)+\sum_{j=1}^{n} d_{i j} f_{j}\left(x_{j}\left(t_{q}-\tau\left(t_{q}, x\left(t_{q}\right)\right)\right)\right)\right]\right) \\
& \leq(\zeta-\epsilon) V_{0}+e^{(\zeta-\epsilon)\left(t_{q}-t_{0}\right)}\left(\sum_{i=1}^{n}-a_{i}\left|x_{i}\left(t_{q}\right)\right|\right) \\
& +e^{(\zeta-\varepsilon)\left(t_{q}-t_{0}\right)} \sum_{i=1}^{n} \sum_{j=1}^{n}\left|b_{i j}\right| \ell_{j}\left|x_{j}\left(t_{q}\right)\right| \\
& +e^{(\zeta-\epsilon)\left(t_{q}-t_{0}\right)}\left(\sum_{i=1}^{n} \sum_{j=1}^{n}\left|d_{i j}\right| \tilde{e}_{j}\left|x_{j}\left(t_{q}-\tau\left(t_{q}, X\left(t_{q}\right)\right)\right)\right|\right) \\
& \leq(\zeta-\epsilon) V_{0} \\
& +e^{(\zeta-\epsilon)\left(t_{q}-t_{0}\right)} \max _{1 \leq i \leq n}\left(-a_{i}+\sum_{j=1}^{n}\left|b_{j i}\right| e_{i}\right)\left\|\mathscr{X}\left(t_{q}\right)\right\| \\
& +e^{(\zeta-\epsilon)\left(t_{q}-t_{0}\right)} \max _{1 \leq i \leq n}\left(\sum_{j=1}^{n}\left|d_{j i}\right| \widetilde{e}_{i}\right)\left\|\mathscr{X}\left(t_{q}-\tau\left(t_{q}, \mathscr{X}\right)\right)\right\| \\
& =\left(\zeta-\epsilon+\pi_{1}\right) V_{0}+e^{(\zeta-\epsilon)\left(t_{q}-\tau\left(t_{q}, X\right)-t_{0}\right)}\left\|\mathscr{X}\left(t_{q}-\tau\left(t_{q}, X\right)\right)\right\| \\
& \times \pi_{2} e^{(\zeta-\epsilon) \tau\left(t_{q}, x\right)} \\
& \leq\left(\zeta-\epsilon+\pi_{1}+\pi_{2} e^{(\zeta-\epsilon) \tau\left(t_{q}, x\right)}\right) V_{0} \\
& =\left(\zeta-\epsilon+\pi_{1}+\pi_{2} e^{(\zeta-\epsilon)\left[\tau\left(t_{q}, x\right)-\tau\left(t_{q}, 0\right)\right]} e^{(\zeta-\epsilon) \tau\left(t_{q}, 0\right)}\right) V_{0} \\
& \leq\left(\zeta-\epsilon+\pi_{1}+\pi_{2} e^{(\zeta-\epsilon) \ell_{\tau}}\left\|x\left(t_{q}\right)\right\|_{1} e^{(\zeta-\epsilon) \tau\left(t_{q}, 0\right)}\right) V_{0} \\
& \leq\left(\zeta-\epsilon+\pi_{1}+\pi_{2} e^{(\zeta-\epsilon)\left(e_{\tau}\left\|x\left(t_{q}\right)\right\|_{1}+\tau_{0}\right)}\right) V_{0} .
\end{aligned}
$$

Together with the definition of $V_{0}, V(t), t_{q}$ and condition (1), we have

$$
\left\|\mathscr{X}\left(t_{q}\right)\right\|_{1}=V\left(t_{q}\right) \leq V_{0}=\|\Psi\|_{\alpha},
$$

and then from (9) and (11), we obtain

$$
\begin{aligned}
& \left.\frac{\mathrm{d}}{\mathrm{d} t}\left(e^{(\zeta-\epsilon)\left(t-t_{0}\right)} V(t)\right)\right|_{t=t_{q}} \\
& \quad \leq\left(\zeta-\epsilon+\pi_{1}+\pi_{2} e^{(\zeta-\epsilon)\left(e_{\tau}\|\Psi\|_{\alpha}+\tau_{0}\right)}\right)\|\Psi\|_{\alpha}<0,
\end{aligned}
$$

which is a contradiction with condition (9), and thus, (10) holds. obtain

Consider the arbitrariness of $\epsilon$, let $\epsilon \longrightarrow 0$, and then we

$$
e^{\zeta\left(t-t_{0}\right)} V(t) \leq V_{0}, \quad \forall t \geq t_{0}
$$

i.e.,

$$
\|\mathscr{X}(t)\|_{1}=V(t) \leq\|\Psi\|_{\alpha} e^{-\zeta\left(t-t_{0}\right)}, \quad \forall t \geq t_{0} .
$$

The reasoning process of Theorem 1 is completed.

Theorem 2. $B$ and $D$ are symmetric matrices; then, under Assumptions 1-4, the zero equilibrium of system (2) is LES if

$$
-2 \mathscr{P}(A)+\lambda_{\max }\left(B^{2}\right)+l_{g}^{2}+\lambda_{\max }\left(D^{2}\right)+l_{f}^{2}<0,
$$

and $\mu=2 \zeta>0$ satisfies

$$
\begin{aligned}
\mu- & 2 \mathscr{P}(A)+\lambda_{\max }\left(B^{2}\right)+l_{g}^{2}+\lambda_{\max }\left(D^{2}\right) \\
& +l_{f}^{2} e^{\mu\left(\ell_{\tau}\|\Psi\|_{\alpha}+\tau_{0}\right)} \leq 0,
\end{aligned}
$$

where $\zeta$ is the Lyapunov exponent.

Proof. Suppose $\mathscr{X}\left(t ; t_{0}, \Psi\right)$ is a solution of system (2) with initial state $\left(t_{0}, \Psi\right)$, where $\Psi \in \mathscr{C}\left(\left[t_{0}-\eta, t_{0}\right], \mathscr{R}^{n}\right)$ and $\Psi \neq 0$. Let $V(t, \mathscr{X})=\left(\|\mathscr{X}(t)\|_{2}\right)^{2}=\mathscr{X}^{T}(t) \mathscr{X}(t)$. For convenience, set $V(t)=V(t, \mathscr{X})$ and $V_{0}=\left\{\sup V(s), s \in\left[t_{0}-\eta, t_{0}\right]\right\}$. Then, for any $\epsilon \in(0, \mu)$, we claim that

$$
e^{(\mu-\epsilon)\left(t-t_{0}\right)} V(t) \leq V_{0}, \quad \forall t \geq t_{0} .
$$

Firstly, when $t=t_{0},(18)$ holds. Next, we prove that (18) holds on $\left(t_{0},+\infty\right)$. In contrast to (18), there are some instants on $\left(t_{0},+\infty\right)$ to make (18) untenable, and then we can find an instant $t_{q} \geq t_{0}$ to satisfy the following three conditions:

(1) $e^{(\mu-\epsilon)\left(t_{q}-t_{0}\right)} V\left(t_{q}\right)=V_{0}$.

(2) $e^{(\mu-\epsilon)\left(t-t_{0}\right)} V(t) \leq V_{0}$, for $\forall t \in\left[t_{0}-\eta, t_{q}\right]$.

(3) There exists a right neighbor of $t_{q}\left(U_{+}^{0}\left(t_{q}, \xi\right)\right)$ such that $\forall t^{\xi} \in U_{+}^{0}\left(t_{q}, \xi\right), e^{(\mu-\epsilon)\left(t^{\xi}-t_{0}\right)} V\left(t^{\xi}\right)>V_{0}$.

On the contrary, by Assumptions 1-4 and Lemma 1, we compute the derivative of $e^{(\mu-\epsilon)\left(t-t_{0}\right)} V(t)$ at time $t_{q}$ : 


$$
\begin{aligned}
& \left.\frac{\mathrm{d}}{\mathrm{d} t}\left(e^{(\mu-\epsilon)\left(t-t_{0}\right)} V(t)\right)\right|_{t=t_{q}} \\
& =(\mu-\epsilon) e^{(\mu-\epsilon)\left(t_{q}-t_{0}\right)} V\left(t_{q}\right)+\left.\left[e^{(\mu-\epsilon)\left(t-t_{0}\right)} \dot{V}(t)\right]\right|_{t=t_{q}} \\
& \leq(\mu-\epsilon) V_{0}+e^{(\mu-\epsilon)\left(t_{q}-t_{0}\right)}\left(2 \mathscr{X}^{T}\left(t_{q}\right) \dot{X}\left(t_{q}\right)\right) \\
& =(\mu-\epsilon) V_{0}+e^{(\mu-\epsilon)\left(t_{q}-t_{0}\right)} \times 2 \mathscr{X}^{T}\left(t_{q}\right) \\
& \times\left[\left(-A \mathscr{X}\left(t_{q}\right)+B g\left(\mathscr{X}\left(t_{q}\right)\right)+D f\left(X\left(t_{q}-\tau\left(t_{q}, \mathscr{X}\right)\right)\right)\right)\right] \\
& =(\mu-\epsilon) V_{0}+e^{(\mu-\epsilon)\left(t_{q}-t_{0}\right)}\left(-2 \mathscr{X}^{T}\left(t_{q}\right) A \mathscr{X}\left(t_{q}\right)\right) \\
& +e^{(\mu-\epsilon)\left(t_{q}-t_{0}\right)}\left(2 \mathscr{X}^{T}\left(t_{q}\right) B g\left(X\left(t_{q}\right)\right)\right) \\
& +e^{(\mu-\epsilon)\left(t_{q}-t_{0}\right)}\left(2 \mathscr{X}^{T}\left(t_{q}\right) D f\left(X\left(t_{q}-\tau\left(t_{q}, \mathscr{X}\right)\right)\right)\right) \\
& \leq(\mu-\epsilon) V_{0}+e^{(\mu-\epsilon)\left(t_{q}-t_{0}\right)}(-2 \mathscr{P}(A)) \mathscr{X}^{T}\left(t_{q}\right) \mathscr{X}\left(t_{q}\right) \\
& +e^{(\mu-\epsilon)\left(t_{q}-t_{0}\right)}\left(\lambda_{\max }\left(B^{2}\right)+l_{g}^{2}\right) \mathscr{X}^{T}\left(t_{q}\right) \mathscr{X}\left(t_{q}\right) \\
& +e^{(\mu-\epsilon)\left(t_{q}-t_{0}\right)} \lambda_{\max }\left(D^{2}\right) \mathscr{X}^{T}\left(t_{q}\right) \mathscr{X}\left(t_{q}\right) \\
& +e^{(\mu-\epsilon)\left(t_{q}-t_{0}\right)} l_{f}^{2} \mathscr{X}^{T}\left(t_{q}-\tau\left(t_{q}, \mathscr{X}\right)\right) \mathscr{X}\left(t_{q}-\tau\left(t_{q}, \mathscr{X}\right)\right) \\
& =\left(\mu-\epsilon-2 \mathscr{P}(A)+\lambda_{\max }\left(B^{2}\right)+l_{g}^{2}+\lambda_{\max }\left(D^{2}\right)\right) V_{0} \\
& +e^{(\mu-\epsilon)\left(t_{q}-\tau\left(t_{q}, X\right)-t_{0}\right)} l_{f}^{2} \mathscr{X}^{T}\left(t_{q}-\tau\left(t_{q}, \mathscr{X}\right)\right) \mathscr{X}\left(t_{q}-\tau\left(t_{q}, \mathscr{X}\right)\right) \\
& \times e^{(\mu-\epsilon) \tau\left(t_{q}, x\right)} \\
& \leq\left(\mu-\epsilon-2 \mathscr{P}(A)+\lambda_{\max }\left(B^{2}\right)+l_{g}^{2}+\lambda_{\max }\left(D^{2}\right)\right) V_{0} \\
& +l_{f}^{2} e^{(\mu-\epsilon) \tau\left(t_{q}, x\right)} V_{0} \\
& =\left(\mu-\epsilon-2 \mathscr{P}(A)+\lambda_{\max }\left(B^{2}\right)+l_{g}^{2}+\lambda_{\max }\left(D^{2}\right)\right) V_{0} \\
& +l_{f}^{2} e^{(\mu-\epsilon)\left[\tau\left(t_{q}, X\right)-\tau\left(t_{q}, 0\right)\right]} e^{(\mu-\epsilon) \tau\left(t_{q}, 0\right)} V_{0} \\
& \leq\left(\mu-\epsilon-2 \mathscr{P}(A)+\lambda_{\max }\left(B^{2}\right)+l_{g}^{2}+\lambda_{\max }\left(D^{2}\right)\right. \\
& \left.+l_{f}^{2} e^{(\mu-\epsilon)\left(e_{\tau}\left\|x\left(t_{q}\right)\right\|_{2}+\tau_{0}\right)}\right) V_{0} .
\end{aligned}
$$

Combining the definition of $V_{0}, V(t), t_{q}$ and condition (1), we have

$$
\left\|\mathscr{X}\left(t_{q}\right)\right\|_{2}=\left(V\left(t_{q}\right)\right)^{1 / 2} \leq\left(V_{0}\right)^{1 / 2}=\|\Psi\|_{\alpha}
$$

and then from (17) and (19), we obtain

$$
\begin{aligned}
& \left.\frac{\mathrm{d}}{\mathrm{d} t}\left(e^{(\mu-\epsilon)\left(t-t_{0}\right)} V(t)\right)\right|_{t=t_{q}} \\
& \leq\left(\mu-\epsilon-2 \mathscr{P}(A)+\lambda_{\max }\left(B^{2}\right)+l_{g}^{2}+\lambda_{\max }\left(D^{2}\right)\right. \\
& \left.\quad+l_{f}^{2} e^{(\mu-\epsilon)\left(\ell_{\tau}\|\Psi\|_{\alpha}+\tau_{0}\right)}\right)\left(\|\Psi\|_{\alpha}\right)^{2}<0 .
\end{aligned}
$$

This is in contradiction with (9), so (18) holds.

Consider the arbitrariness of $\epsilon$, let $\epsilon \longrightarrow 0$, and then we could be capable of getting

$$
e^{\mu\left(t-t_{0}\right)} V(t) \leq V_{0}, \quad \forall t \geq t_{0},
$$

i.e.,

$$
\|\mathscr{X}(t)\|_{2}=(V(t))^{1 / 2} \leq\|\Psi\|_{\alpha} e^{-(\mu / 2)\left(t-t_{0}\right)}, \quad \forall t \geq t_{0} .
$$

The reasoning process of Theorem 2 is completed.

Remark 2. In Theorem 2, by taking the value of $₫$ in Lemma 1 to be 1 , we can get the following inequality:

$$
\begin{aligned}
& 2 X^{T}\left(t_{q}\right) B g\left(X\left(t_{q}\right)\right)=\mathscr{X}^{T}\left(t_{q}\right) B g\left(X\left(t_{q}\right)\right)+\mathscr{X}^{T}\left(t_{q}\right) B g\left(X\left(t_{q}\right)\right) \\
& =\mathscr{X}^{T}\left(t_{q}\right) B^{T} g\left(x\left(t_{q}\right)\right)+g^{T}\left(X\left(t_{q}\right)\right) B \mathscr{X}\left(t_{q}\right) \\
& =\left(B X\left(t_{q}\right)\right)^{T} g\left(x\left(t_{q}\right)\right)+g^{T}\left(X\left(t_{q}\right)\right)\left(B X\left(t_{q}\right)\right) \\
& \leq\left(B X\left(t_{q}\right)\right)^{T}\left(B X\left(t_{q}\right)\right)+g^{T}\left(X\left(t_{q}\right)\right) g\left(x\left(t_{q}\right)\right) \\
& =\mathscr{X}^{T}\left(t_{q}\right) B^{T} B \mathscr{X}\left(t_{q}\right)+g^{T}\left(X\left(t_{q}\right)\right) g\left(X\left(t_{q}\right)\right) \\
& =X^{T}\left(t_{q}\right) B^{2} X\left(t_{q}\right)+g^{T}\left(X\left(t_{q}\right)\right) g\left(X\left(t_{q}\right)\right) \\
& \leq \lambda_{\max }\left(B^{2}\right) \mathscr{X}^{T}\left(t_{q}\right) \mathscr{X}\left(t_{q}\right)+l_{g}^{2} \mathscr{X}^{T}\left(t_{q}\right) \mathscr{X}\left(t_{q}\right) \\
& =\left(\lambda_{\max }\left(B^{2}\right)+l_{g}^{2}\right) \mathscr{X}^{T}\left(t_{q}\right) \cdot X\left(t_{q}\right) \text {. }
\end{aligned}
$$

We give a particular case of system (2), considering the following one-dimensional system:

$$
\begin{aligned}
\dot{x}(t) & =a x(t)+b g(x(t))+d f(x(t-\tau(t, x))), t \geq 0, \\
\tau(t, x) & =\delta+\lambda x(t),
\end{aligned}
$$

where $a, b, d, \delta, \lambda \in \mathscr{R}$.

Corollary 1. If system (25) satisfies the following conditions,

(1) $f(0)=0, g(0)=0 . g(\cdot), f(\cdot)$ are locally Lipschitz continuous functions whose Lipschitz constants are $l_{1}, l_{2}$, respectively,

(2) $a<0$ and $a+|b| l_{1}+|d| l_{2}<0$,

(3) $\delta>0$, and the initial condition of system (25) satisfies $|\psi(\cdot)| \leq M<(\delta /|\lambda|)$, where $|\psi(\cdot)|=\max _{-\eta \leq t \leq 0}|\psi(\cdot)|$, then we can obtain

$$
|x(t)| \leq M e^{-\zeta t}, \quad t \geq 0,
$$


where $x(t)=x(t ; 0, \psi)$ represents the trajectory of system (25) with initial state $(0, \psi)$, and Lyapunov exponent $\zeta>0$ satisfies

$$
\zeta+a+|b| l_{1}+|d| l_{2} e^{\zeta(|\lambda| M+\delta)} \leq 0 .
$$

Proof. To prove Corollary 1, we only need to prove that Corollary 1 satisfies Assumptions 1-4. Obviously, from condition (1), we can know that system (25) satisfies Assumptions 1 and 2. Next, we prove that system (25) satisfies Assumptions 3 and 4.

We assume that $x(t ; 0, \psi)$ is a trajectory of system (25) with initial value $(0, \psi)$, where $\psi \neq 0$. From $\tau(t, x)=\delta+$ $\lambda x(t)$, we could know that $\tau(t, x)$ is continuous about $t$. Owing to $|\psi| \leq M$ and $M<(\delta /|\lambda|)$, there exists $\rho>0$ such that $\forall t \in[0, \rho]$ and $\tau(t, x) \geq 0$. In the circumstances, set $V(t)=|x(t)|$. Next, under conditions (1)-(3) and by Theorem 1 , we get $|x(t)| \leq M e^{-\zeta t}$, for $\forall t \in[0, \rho]$, where Lyapunov exponent $\zeta$ satisfies (27).

Then, for $\forall t \in[0,+\infty)$, we declare that $\tau(t, x) \geq 0$. In contrast to the claim, there must be some instant on $(\rho,+\infty)$ to make $\tau(t, x(t))<0$. Then, we could find an instant $t_{q} \in[\rho,+\infty)$ to meet the following three conditions:

(1) $\tau\left(t_{q}, x\left(t_{q}\right)\right)=\delta+\lambda x\left(t_{q}\right)=0$.

(2) $\forall t \in\left[0, t_{q}\right], \tau(t, x(t)) \geq 0$.

(3) There exists a right neighbor of $t_{q}\left(U_{+}^{0}\left(t_{q}, \xi\right)\right)$ such that $\tau\left(t^{\xi}, x\left(t^{\xi}\right)\right)<0$, for any $t^{\xi} \in U_{+}^{0}\left(t_{q}, \xi\right)$.

It follows from Theorem 1 , for any $t \in\left[0, t_{q}\right]$, we have $|x(t)| \leq M e^{-\zeta t}$, which indicates that $\left|x\left(t_{q}\right)\right| \leq M e^{-\zeta t_{q}}<$ $(\delta /|\lambda|)$. On the basis of the continuity of $x(t)$, there exists $\xi^{0}>0$ such that $|x(t)|<(\delta /|\lambda|)$, for any $t \in\left[t_{q}, t_{q}+\xi^{0}\right)$. It means that $\forall t \in\left[t_{q}, t_{q}+\xi^{0}\right)$, and $\tau(t, x(t))=\delta+\lambda x(t)>0$ holds, which contradicts with (9); therefore, $\forall t \in[0,+\infty)$ and $\tau(t, x(t)) \geq 0$. It ulteriorly indicates that Assumptions 3 and 4 are all satisfied. Therefore, it can be known from Theorem 1 that Corollary 1 is valid. The proof is completed.

Remark 3. Actually, in Theorem 1, when $\pi_{1}+\pi_{2}<0$, we can definitely get $\zeta+\pi_{1}+\pi_{2} e^{\zeta\left(\ell_{\tau}\|\Psi\|+\tau_{0}\right)} \leq 0$ as long as $\zeta$ is small enough. Theorem 2 has a similar conclusion.

Remark 4. In Theorem 1, by taking $t_{q}-\tau\left(t_{q}, \mathscr{X}\right)$ as a whole and then from condition (2), we are able to get $e^{(\zeta-\epsilon)\left(t_{q}-\tau\left(t_{q}, X\right)-t_{0}\right)}\left\|\mathscr{X}\left(t_{q}-\tau\left(t_{q}, \mathscr{X}\right)\right)\right\| \leq V_{0}$. Theorem 2 has a similar conclusion.

Remark 5. The proofs of Theorems 1 and 2 also provide an estimate of the locally exponentially convergent rate $\zeta$ which could be obtained by solving transcendental equation (9) or (17).

Remark 6. Remarkably, the Lyapunov exponent in Theorems 1 and 2 is state-dependent, so only when the initial value is bounded can we find common $\zeta$ to make $e^{(\zeta-\epsilon)} V(t) \leq V(0)$. Furthermore, due to the effect of SDSD, the results in our paper are local features, not global features.

\section{Illustrative Examples}

To prove the effectiveness of our results, two numerical examples will be given in this section.

Example 1. Consider a 2-dimensional neural network with SDSD, which is described by

$$
\begin{aligned}
\left(\begin{array}{l}
\dot{x}_{1}(t) \\
\dot{x}_{2}(t)
\end{array}\right)= & -\left(\begin{array}{ll}
1 & 0 \\
0 & 1
\end{array}\right)\left(\begin{array}{l}
x_{1}(t) \\
x_{2}(t)
\end{array}\right) \\
& +\left(\begin{array}{ll}
0.25 & 0.25 \\
0.02 & 0.01
\end{array}\right)\left(\begin{array}{l}
g_{1}\left(x_{1}(t)\right) \\
g_{2}\left(x_{2}(t)\right)
\end{array}\right) \\
& +\left(\begin{array}{cc}
0.3 & 0.2 \\
0.01 & 0.02
\end{array}\right)\left(\begin{array}{l}
f_{1}\left(x_{1}(t-\tau(t, \mathscr{X}))\right) \\
f_{2}\left(x_{2}(t-\tau(t, \mathscr{X}))\right)
\end{array}\right),
\end{aligned}
$$

where $t_{0}=0$ and

$$
\begin{aligned}
g_{i}(\cdot) & =\left|x_{i}(t)+1\right|+\left|x_{i}(t)-1\right|, \quad i=1,2, \\
f_{i}(\cdot) & =\sin \left(x_{i}\left(t-\left|\sin \left(x_{1}(t)+x_{2}(t)\right)\right|\right)\right), \quad i=1,2, \\
\tau(t, \mathscr{X}) & =\left|\sin \left(x_{1}(t)+x_{2}(t)\right)\right| .
\end{aligned}
$$

Evidently, $\quad \ell_{1}=\ell_{2}=2, \widetilde{\ell}_{1}=\widetilde{\ell}_{2}=1, \ell_{\tau}=1, \tau(t, 0)=$ $0, \tau(t, \mathscr{X}) \in[0,1]$. By calculating,

$$
\begin{aligned}
& \pi_{1}=\max _{1 \leq i \leq n}\left(-a_{i}+\sum_{j=1}^{n}\left|b_{j i}\right| \ell_{i}\right)=-0.46, \\
& \pi_{2}=\max _{1 \leq i \leq n} \sum_{j=1}^{n}\left|d_{j i}\right| \widetilde{\ell}_{i}=0.31 .
\end{aligned}
$$

Then, from Theorem 1, system (28) is LES. The trajectories of the solution from a random initial value are shown in Figure 1. As shown in Figure 1, $x_{1}(t)$ and $x_{2}(t)$ in neural network model (28) are convergent. Figure 2 shows the phase diagram of system (28) evolving with time.

Example 2. Consider another 2-dimensional neural network with SDSD, which is depicted by

$$
\begin{aligned}
\left(\begin{array}{l}
\dot{x}_{1}(t) \\
\dot{x}_{2}(t)
\end{array}\right)= & -\left(\begin{array}{ll}
2 & 0 \\
0 & 2
\end{array}\right)\left(\begin{array}{l}
x_{1}(t) \\
x_{2}(t)
\end{array}\right) \\
& +\left(\begin{array}{ll}
0.5 & 0.2 \\
0.2 & 0.5
\end{array}\right)\left(\begin{array}{l}
g_{1}\left(x_{1}(t)\right) \\
g_{2}\left(x_{2}(t)\right)
\end{array}\right) \\
& +\left(\begin{array}{ll}
0.3 & 0.2 \\
0.2 & 0.3
\end{array}\right)\left(\begin{array}{l}
f_{1}\left(x_{1}(t-(t, X))\right) \\
f_{2}\left(x_{2}(t-(t, \mathscr{X}))\right)
\end{array}\right),
\end{aligned}
$$

where $t_{0}=0$ and 


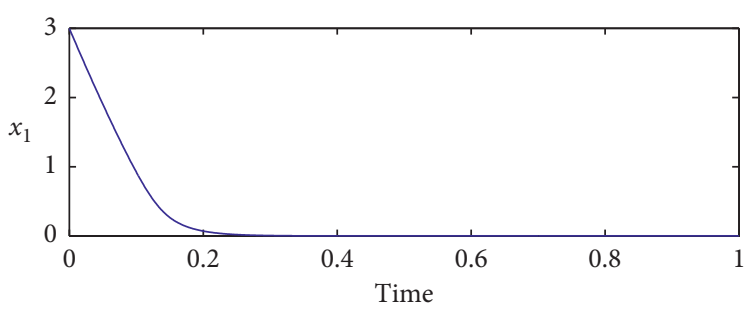

(a)

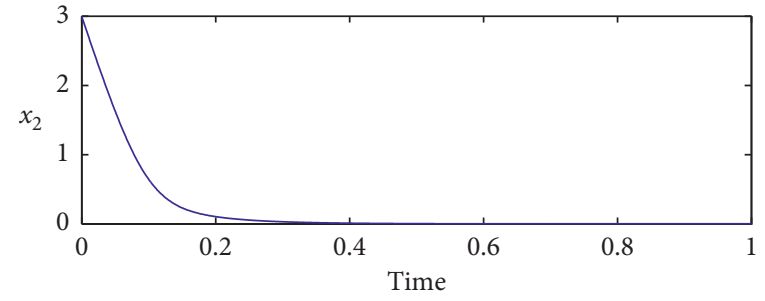

(b)

Figure 1: Transient behavior of (a) $x_{1}(t)$ and (b) $x_{2}(t)$ in system (28).

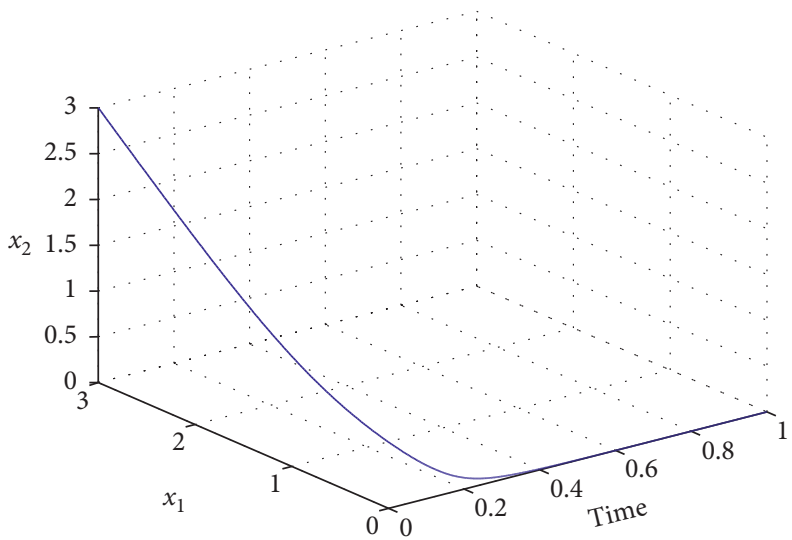

Figure 2: Transient behavior of $\left(x_{1}(t), x_{2}(t)\right)$ in system (28).

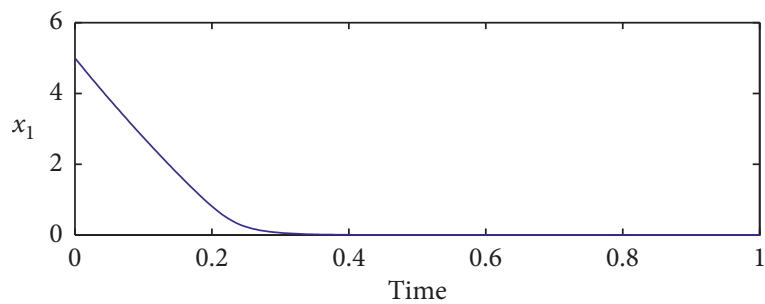

(a)

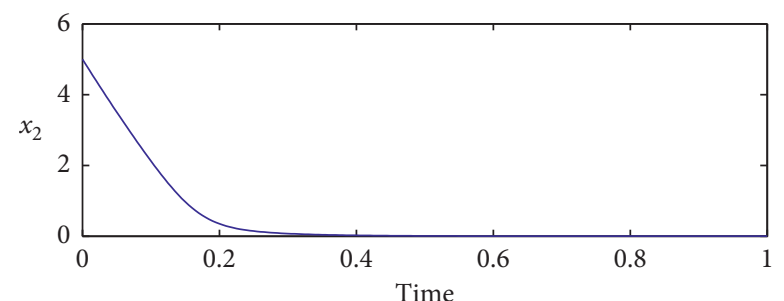

(b)

Figure 3: Transient behavior of (a) $x_{1}(t)$ and (b) $x_{2}(t)$ in system (31).

$$
\begin{aligned}
g_{i}(\cdot) & =\frac{\cos \left(x_{i}(t)+(\pi / 2)\right)}{2}, \quad i=1,2, \\
f_{i}(\cdot) & =\tanh \left(x_{i}\left(t-\left|\sin \left(x_{1}(t)+x_{2}(t)\right)\right|\right)\right), \quad i=1,2, \\
\tau(t, \mathscr{X}) & =\left|\sin \left(x_{1}(t)+x_{2}(t)\right)\right| .
\end{aligned}
$$

Obviously, $\quad l_{g}=1 / 2, l_{f}=1, \ell_{\tau}=1, \tau(t, 0)=0,(t, \mathscr{X}) \epsilon$ $[0,1], \mathscr{P}(A)=2$. By computing, $\quad \lambda_{\text {max }}\left(B^{2}\right)=0.49, \lambda_{\max }$ $\left(C^{2}\right)=0.25$, and $-2 \mathscr{P}(A)+l_{g}^{2}+l_{f}^{2}+\lambda\left(B^{2}\right)+\lambda\left(C^{2}\right)=-4+$ $(1 / 4)+1+0.49+0.25=-2.01<0$. Then, from Theorem 2 , system (31) is LES. The trajectories of the solution from a random initial value are shown in Figure 3. As shown in Figure 3, $x_{1}(t)$ and $x_{2}(t)$ in neural network model (31) are

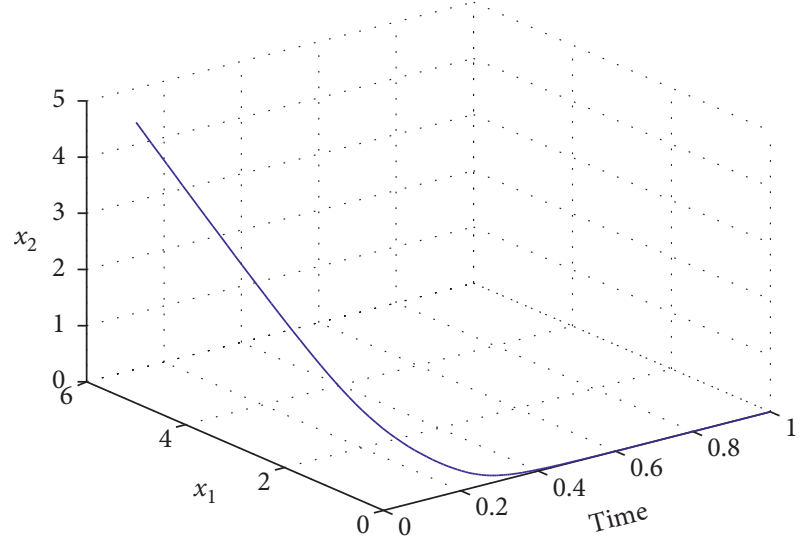

Figure 4: Transient behavior of $\left(x_{1}(t), x_{2}(t)\right)$ in system (31). 
convergent. Figure 4 shows the phase diagram of system (31) evolving with time.

\section{Concluding Remarks}

We devote to resolve the problem of local dynamic property for neural networks with SDSD in this paper. Through pure analysis method and technique of reduction to absurdity, we obtain a certain number of sufficient conditions for local exponential stability of neural networks with SDSD. Based on our results, we know that the Lyapunov exponent relies on the state on account of the effect of SDSD. It also indicates that the exponential stability results derived are local rather than global. Consequently, we can take the global dynamics as a topic in the prospective research. In addition, we can also develop SDSD system methods, for instance, eventtriggered control.

\section{Data Availability}

No data were used to support this study.

\section{Conflicts of Interest}

The authors declare that there are no conflicts of interest regarding the publication of this paper.

\section{Acknowledgments}

The work of the first author for master student Yue Chen was done under the direction of Assistant Professor Jin-E Zhang. This work was supported by the Natural Science Foundation of China under Grants 61976084 and 61773152.

\section{References}

[1] L. Teng and D. Xu, "Global attracting set for non-autonomous neutral type neural networks with distributed delays," Neurocomputing, vol. 94, pp. 64-67, 2012.

[2] J. Cao and J. Wang, "Global exponential stability and periodicity of recurrent neural networks with time delays," IEEE Transactions on Circuits and Systems I: Regular Papers, vol. 52, no. 5, pp. 920-931, 2005.

[3] Z. Yang, J. Peng, and Y. Liu, “Adaptive neural network force tracking impedance control for uncertain robotic manipulator based on nonlinear velocity observer," Neurocomputing, vol. 331, pp. 263-280, 2019.

[4] H.-G. Han, Y. Li, Y.-N. Guo, and J.-F. Qiao, "A soft computing method to predict sludge volume index based on a recurrent self-organizing neural network," Applied Soft Computing, vol. 38, pp. 477-486, 2016.

[5] W. S. Chen and J. M. Li, "Adaptive neural network tracking control for a class of unknown nonlinear time-delay systems," Journal of Systems Engineering and Electronics, vol. 17, no. 3, pp. 611-618, 2006.

[6] T. Zan, Z. Liu, H. Wang, M. Wang, and X. Gao, "Control chart pattern recognition using the convolutional neural network," Journal of Intelligent Manufacturing, vol. 31, no. 3, pp. 703716, 2020.

[7] V. Narayanan, S. Jagannathan, and K. Ramkumar, "Eventsampled output feedback control of robot manipulators using neural networks," IEEE Transactions on Neural Networks and Learning Systems, vol. 30, no. 6, pp. 1651-1658, 2019.

[8] B. L. Chen, H. D. Li, W. Q. Luo, and J. W. Huang, "Image processing operations identification via convolutional neural network," Science China Information Sciences, vol. 63, no. 3, p. 139109, 2020.

[9] M. M. Van Hulle and J. Larsen, "Neural networks in signal processing," Neurocomputing, vol. 69, no. 1-3, pp. 1-2, 2005.

[10] Y. Hayakawa and K. Nakajima, "Design of the inverse function delayed neural network for solving combinatorial optimization problems," IEEE Transactions on Neural Networks, vol. 21, no. 2, pp. 224-237, 2010.

[11] Z. Zeng and J. Wang, "Analysis and design of associative memories based on recurrent neural networks with linear saturation activation functions and time-varying delays," Neural Computation, vol. 19, no. 8, pp. 2149-2182, 2007.

[12] Z. Li, L. Liu, and Q. Zhu, "Mean-square exponential input-tostate stability of delayed Cohen-Grossberg neural networks with Markovian switching based on vector Lyapunov functions," Neural Networks, vol. 84, pp. 39-46, 2016.

[13] A. Wu and Z. Zeng, "Lagrange stability of memristive neural networks with discrete and distributed delays," IEEE Transactions on Neural Networks and Learning Systems, vol. 25, no. 4, pp. 690-703, 2014.

[14] T. Ensari and S. Arik, "Global stability of a class of neural networks with time-varying delay," IEEE Transactions on Circuits and Systems II: Express Briefs, vol. 52, no. 3, pp. 126-130, 2005.

[15] L. Li, Y. Yang, and F. Wang, "The sampled-data exponential stability of BAM with distributed leakage delays," Neural Processing Letters, vol. 46, no. 2, pp. 537-547, 2017.

[16] H. Liu, L. Zhao, Z. Zhang, and Y. Ou, "Stochastic stability of markovian jumping Hopfield neural networks with constant and distributed delays," Neurocomputing, vol. 72, no. 16-18, pp. 3669-3674, 2009.

[17] C. Zheng, N. Li, and J. Cao, "Matrix measure based stability criteria for high-order neural networks with proportional delay," Neurocomputing, vol. 149, pp. 1149-1154, 2015.

[18] J. Y. Zhang, "Global stability analysis in cellular neural networks with unbounded time delays," Applied Mathematics and Mechanics, vol. 25, no. 6, pp. 686-693, 2004.

[19] R. Gambell, "Birds and Mammals-Antarctic whales," Edited by W. Bonner and D. Walton, Eds., pp. 223-241, Pergamon Press, New York, NY, USA, 1985.

[20] L. Liu, L. Zhu, and D. Yang, "Modeling and simulation of the car-truck heterogeneous traffic flow based on a nonlinear carfollowing model," Applied Mathematics and Computation, vol. 273, pp. 706-717, 2016.

[21] S. Yu and Z. Shi, "An improved car-following model considering relative velocity fluctuation," Communications in Nonlinear Science and Numerical Simulation, vol. 36, pp. 319-326, 2016.

[22] P. Getto and M. Waurick, "A differential equation with statedependent delay from cell population biology," Journal of Differential Equations, vol. 260, no. 7, pp. 6176-6200, 2016.

[23] F. Hartung, "Linearized stability in periodic functional differential equations with state-dependent delays," Journal of Computational and Applied Mathematics, vol. 174, no. 2, pp. 201-211, 2005.

[24] I. Györi and F. Hartung, "Exponential stability of a statedependent delay system," Discrete \& Continuous Dynamical Systems- $A$, vol. 18, no. 4, pp. 773-791, 2007.

[25] C. Fiter and E. Fridman, "Stability of piecewise affine systems with state-dependent delay, and application to congestion control," in 
Proceedings of the 52nd IEEE Annual Conference on Decision and Control, pp. 1572-1577, Florence, Italy, December 2013.

[26] X. Li and J. Wu, "Stability of nonlinear differential systems with state-dependent delayed impulses," Automatica, vol. 64, pp. 63-69, 2016.

[27] X. Li and X. Yang, "Lyapunov stability analysis for nonlinear systems with state-dependent state delay," Automatica, vol. 112, p. 108674, 2020.

[28] C. Zhou, X. Zeng, J. Yu, and H. Jiang, "A unified associative memory model based on external inputs of continuous recurrent neural networks," Neurocomputing, vol. 186, pp. 44$53,2016$.

[29] L. Liu, X. Ding, W. Zhou, and X. Li, "Global mean square exponential stability and stabilization of uncertain switched delay systems with Lévy noise and flexible switching signals," Journal of the Franklin Institute, vol. 356, no. 18, pp. 11520$11545,2019$. 